\title{
El problema mente-cuerpo. Un ensayo de antropología wittgensteiniana
}

\author{
Carlos Rodríguez Sutil \\ Universidad Complutense de Madrid

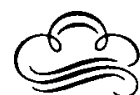 \\ Resumen
}

\begin{abstract}
Este articulo representa un intento por resolver el problema antropológico clásico, especialmente planteado por Descartes, de la relación mente-cuerpo, que tantos problemas ba causado en psicologí. Para ello nos inspiramos en la crítica de las entidades internas que realiza el segundo Wittgenstein mediante su Argumento del Lenguaje Privado. Después aplicamos dicha crítica a los reduccionismos verificacionistas en psicología, conductismo y fisiologismo, asi como a la teoría de James-Lange sobre las emociones. El error de estas posturas consiste en partir del dualismo cartesiano y de su perspectiva egocéntrica como supuesto implícito.
\end{abstract}

Palabras clave: Mente y cuerpo, reduccionismo, conductismo, fisiologismo, emociones, James-Lange, Wittgenstein.

\begin{abstract}
This article strives to offer a solution to the classical antbropological problem, especially stated by Descarte, concerning the relationsbip between mind and body. This problem bas caused great confusion in Psychology. In order to attain that goal we recur to the criticism of internal entities by Wittgenstein and his Private Language Argument. Subsequently we refer this criticism to the psychological verificational reductionisms: behaviorism and physiologism; and to the James-Lange theory of emotions. The mistake shared by these approaches is the implicit presupposition of Cartesian dualism and its egocentric perspective.
\end{abstract}

Key words: Mind and Body, Reductionism, Behaviorism, Physiologism, Emotions, JamesLange, Wittgenstein.

Dirección del autor: Departamento de Personalidad, Evaluación y Tratamiento. Facultad de Psicología de la Universidad Complutense. Campus de Somosaguas. 28023 - MADRID. 


\section{INTRODUCCION}

Hace algunos años el conocido filósofo de la ciencia Frederick Suppe (1974, p. 38) deploraba el hecho de que con frecuencia los científicos siguen manteniendo posiciones filosóficas mucho tiempo después de que han caído en descrédito. Recurre al ejemplo del conductismo radical de Skinner, quien se aferra a las definiciones operacionales cuando los expertos ya habían mostrado su debilidad.

En psicología y, en general, en las ciencias sociales, no es frecuente que una línea de investigación se abandone por completo aunque sus fundamentos epistemológicos hayan sido cuestionados, sino que perviven y coexisten, de forma no siempre pacífica, las más diversas escuelas. Las razones de esto son sin duda complejas y habría que buscarlas, contestando a Suppe, no en el distanciamiento entre filosofía y psicología sino, bien al contrario, en su mutua imbricación. Como reconocía el psicólogo cognitivo norteamericano Jerome Bruner (1983), en un interesante ensayo autobiográfico, la psicología de la mente nunca podrá estar libre de la filosofía de la mente.

El presente ensayo intenta clarificar las bases conceptuales de un problema antropológico de gran importancia, como es el de la relación mente-cuerpo, motivo histórico de múltiples confusiones. Confiamos en que para resolver, o para «disolver», dichas confusiones, es sumamente útil recurrir a la crítica de las entidades internas que nos proporciona Wittgenstein con su Argumento del Lenguaje Privado (ALP), argumento que ya hemos expuesto detenidamente en otro lugar (Rodríguez Sutil, en prensa). Wittgenstein, no obstante, no es propiamente un psicólogo, ni propone un modelo teórico alternativo, sino que se dedica a desarraigar algunos mitos muy persistentes en el pensamiento occidental. Después examinaremos cómo se aplica esta labor a los reduccionismos, tanto conductista como fisiológico y a la teoría de James-Lange sobre las emociones.

Es habitual distinguir la obra del primer y del segundo Wittgenstein, representada en especial por el Tractatus (1921) y por las Philosophical Investigations (1952), respectivamente. Aquí nos ocuparemos sobre todo de la obra de madurez. La inspiración ética de los jóvenes intelectuales vieneses - encabezados por el periodista Karl Kraus - no le habría de abandonar el resto de su vida, y dirigirá, de manera implícita, su crítica a las formas dominantes de pensamiento (Cf. Janik y Toulmin, 1973). Wittgenstein se encuentra con un pensamiento enfermo y advierte en varios lugares la semejanza entre su método filosófico y una técnica curativa. En las Philosophical Investigations (PI, I, 255) ${ }^{1}$ se dice que el filósofo es alguien que debe tratar una pregunta como una enfermedad.

\section{CARTESIANISMO Y PSICOLOGIA}

La crítica de Wittgenstein a los conceptos psicológicos no es comprensible sin una exposición, al menos sucinta, de su radical cuestionamiento de las dos sustancias cartesianas: pensamiento y extensión, o mente y materia; consideradas, en definitiva, como dos espacios herméticamente cerrados con una conexión causal problemática: el paralelismo psicofísico. Las dificultades del paralelismo no han sido resueltas ni por el idealismo (solipsista) ni por el empirismo (materialista), ni por las múltiples posturas intermedias. En las Philosopbical Investigations se cumple, de manera radical, merced al ALP, la destrucción del supuesto básico del dualismo cartesiano: la existencia de un lenguaje priva- 


\section{9}

do, anterior al aprendizaje de la lengua materna. Wittgenstein eligió ahí como motivo un párrafo de las Confesiones de San Agustín, pero dicho supuesto, como leemos en García Suárez (1976, pp. 21-23), está después presente en racionalistas, empiristas, fisicistas y dualistas cartesianos, continentales y angloparlantes. La razón de presencia tan diversa radica en la adopción, confesa o tácita, no tanto de una doctrina filosófica concreta, como de un punto de partida. Se trata de la perspectiva egocéntrica, inaugurada oficialmente por Descartes, y seguida por el empirismo clásico (idea) y contemporáneo sense-data.

Nuestro filósofo viene a decir, siguiendo la explicación del británico Kenny (1972, p. 161) uno de sus comentadores más perspicaces, que los defensores del lenguaje privado cometen dos errores: a) creer que la experiencia es privada y b) creer que las palabras pueden adquirir significado mediante definiciones ostensivas (privadas). Recordemos que una definición ostensiva es la que supone, ingenuamente, que podemos establecer una relación directa entre el signo y el objeto sin tener en cuenta el sistema del lenguaje, tomando esa relación como unidad básica del conocimiento humano. Podríamos afirmar, comenta Kenny, que el dolor es privado en cuanto a su conocimiento o en cuanto a su posesión. La primera posibilidad recibe el nombre de «incomunicabilidad», y el de «inalienabilidad», la segunda. Respecto a la incomunicabilidad, Wittgenstein escribió:

¿Hasta qué punto son mis sensaciones privadas?

- Bueno, sólo yo puedo saber si realmente tengo dolor; el otro sólo puede presumirlo. - Esto es en cierto modo falso y en otro un sinsentido (...)

Los demás saben muy frecuentemente cuándo tengo dolor (...). De mí no puede decirse en absoluto (excepto quizá en broma) que sé que tengo dolor. (PI, I, 246)

De alguna manera es cierto que una persona no puede saber si otra tiene dolores, pero no porque en realidad no se sepa, sino porque no tiene sentido decir que se sabe o no. El enunciado no es comparable con una afirmación de facto, como «es posible para un ser humano cruzar a nado el Atlántico», sino con otra de corte lógico o gramatical, es decir, perteneciente al modo de representación, como «no hay meta en una carrera de resistencia». En cuanto a la inalienabilidad dice:

«Otro no puede tener mis dolores» — ¿Qué son mis dolores? ¿Qué cuenta aquí como criterio de identidad? (...) «iPero otro no puede sin embargo tener ESTE dolor!» - La respuesta a esto es que no se define ningún criterio de identidad mediante la acentuación enfática de la palabra «éste». (PI, I, 253)

Las Philosopbical Investigations demuestran la imposibilidad lógica de una definición ostensiva interna (PI, I, 258). La estructura gramatical objeto/designación (en alemán Gegenstand/Bezeichnung) no es adecuada para conceptualizar las sensaciones (PI, I, 293). Esta es una idea que puede sorprender porque va en contra de nuestros hábitos lingüísticos cotidianos $y$, por tanto, de nuestra tendencia de pensamiento. Lo cierto es que no existe un objeto interno «dolor» al que corresponda la denominación «dolor». La palabra «dolor» es en ocasiones el sustituto y habitualmente el acompañante de la conducta primitiva de dolor. Si los seres humanos no manifestaran dolor no se le podría enseñar a un niño la expresión «dolor de muelas», pero cuando damos un nombre al dolor presuponemos la gramática de la palabra «dolor» (PI, I, 257). No es que no exista la mentira, pero para mentir tenemos que aprender primero a hablar (PI, I, 249, 250). Imaginemos el caso (Cf. PI, I, 293) de que cada persona tuviera una caja en la que se supone que guarda algo que llamamos «escarabajo», pero nadie puede 
mirar en la caja de otra persona y sólo sabe de qué se trata por la visión de su propio escarabajo (definición ostensiva interna). Peri si la palabra «escarabajo» tuviera un uso no habría de confundirse con la designación de una cosa, la cosa podría incluso no existir, ni siquiera sería un algo. Las palabras no tienen significado porque se correspondan con algún objeto sino porque forman parte de algún juego de lenguaje, entendido como sistema.

\section{PENSAR CON LA CABEZA}

Una de las ideas más peligrosas para un filósofo, escribe Wittgenstein en los Zettel $(Z, 605,606)$, es que pensamos en nuestras cabezas, en un espacio completamente cerrado, oculto. Esta confusión procede de lo que el filósofo de Oxford Gilbert Ryle (1949) denominó «error categorial». Tomemos el ejemplo del visitante que acude a la universidad y, después de haberle mostrado las aulas, laboratorios, bibliotecas, etc., pregunta dónde exactamente se encuentra «la» universidad. «Mental» $\mathrm{y}$ «material» pertenecen a distintas categorías lógicas; el error categorial consiste en buscar un espacio material donde se localice lo mental, la res cogitans cartesiana. Una vez que se le atribuye ese espacio - la caja craneana en nuestra cultura, no así en otras - se dota a lo mental de características similares a lo material (fenoménico). Comentaba Wittgenstein a sus alumnos en el curso 33-34 que tal vez la razón por la que nos inclinamos a hablar de la cabeza como del lugar de nuestros pensamientos es por la existencia de palabras como «pensar» y «pensamiento» junto a las palabras que denotan actividades (corporales), tales como escribir, hablar, etc. La existencia de los últimos verbos nos hace buscar una actividad, diferente de éstas, pero análoga a ellas, que corresponda a la palabra «pensar»: «Cuando las palabras tienen prima facie en nuestro lenguaje ordinario gramáticas análogas, nos inclinamos a intentar interpretarlas análogamente; es decir, tratamos de hacer valer la analogía en todos los campos» (BIB, p. 7; pp. 33-34 de la traducción castellana).

Wittgenstein ataca el dualismo pero, como advierte David Pole (1958, p. 149 y sig.), no por ello hay que tomarle por un «monista». No niega que los términos mentales tengan un uso, pero intenta evitar la imagen de interioridad que se les asocia (PI, II, p. 223). Este «rechazo» de la interioridad lleva a que se identifique a nuestro filósofo con el conductismo.

\section{VERIFICACIONISMO Y CONDUCTISMO}

A continuación no nos ocuparemos tanto de las teorías de estímulo y respuesta, o de conducta y refuerzo, como del punto de vista filosófico (conductismo lógico) que, en sus dos formas, defiende o bien que los términos mentales pueden ser traducidos completamente en términos de la conducta corporal y de las circunstancias físicas en que se produce (conductismo radical), o bien que, aun existiendo eventos mentales no traducibles, el campo de la ciencia psicológica sólo puede corresponder a la conducta corporal y sus circunstancias físicas (conductismo metodológico).

El conductismo hunde sus raíces en el positivismo lógico vienés y, más en concreto, en la propuesta de Rudolf Carnap (1932-33) de una psicología en lenguaje fisicalista.

Los filósofos del Círculo de Viena deseaban una filosofía científica, libre de las especulaciones metafísicas del pasado. Consideraban que toda proposi- 
ción cae dentro de dos categorías, o es lógica y, por tanto, carece de significado (es una tautología), o es empírica, en cuyo caso debe ser verificable. Toda proposición que no entre en esas dos categorías es un sinsentido (Kraft, 1950; Ursom, 1956). El operacionalismo, u operativismo, que tanto repercutió en psicologías, puede ser considerado como una forma del «principio de verificación» (Cf. Hempel, 1954).

Sin pretender relatar aquí pormenorizadamente los avatares del verificacionismo, debemos señalar que pronto se apreció la imposibilidad de mantener el principio en su forma original: con él se eliminaban como sinsentidos muchas proposiciones de la ciencia que no eran fácilmente verificables. Esto dio paso a las versiones débiles del principio. Muchas veces se ha pretendido emparentar a Wittgenstein con el verificacionismo del Círculo de Viena y, por ende, se le ha considerado un conductista (lógico). En cuanto a lo primero, señalemos que, en cualquier caso, si nuestro filósofo defendió el principio de verificación, sólo fue en una versión débil (Cf. p. e. PR, 282), versión que más parecido semeja tener con la forma de verificación propia del pragmatismo, en concreto, de William James (1907). En opinión de James la verdad no es una propiedad estática de las ideas, sino que las ideas verdaderas son aquellas que podemos hacer válidas, y falsas las que no. La verdad es algo que acontece a la idea y, lejos de ser un fin en sí misma nos guía para la obtención de otras satisfacciones vitales. Excuse el lector que no demos un tratamiento exhaustivo, por razones de espacio, al asunto del verificacionismo en Wittgenstein.

En cuanto a su supuesto conductismo, se ha recurrido para justificarlo a la noción de criterio que nos legó (Cf. Chihara y Fodor, 1960). Wittgenstein desarrolla las nociones de criterio y se sintoma en el contexto de su ALP: los «procesos privados» requieren «criterios externos» (PI, I, 579, 580). El descenso del barómetro es un «síntoma» de lluvia, el asomarnos por la ventana y ver caer gotas es un «criterio» (PI, I, 354). En otro orden de cosas, el significado del término «lluvia» no se enseña señalando un barómetro. Los síntomas son acontecimientos que ocurren en relación temporal con cierto fenómeno, pero que no sirven de criterio. Pues el que algo sea criterio de $\mathrm{X}$ no es cuestión de experiencia sino de definición. Un proceso en el cerebro de un hombre o en su laringe puede ser un síntoma de que está viendo algo rojo, pero el criterio es lo que dice y hace (PI, I, 376, 377).

El criterio de que yo recuerdo el ejemplar correcto de la sensación «S» sólo puede ser externo, y está integrado en el aprendizaje social que me ha permitido identificar mi comportamiento (primitivo) con una palabra y, en algún caso, sustituirlo, no sólo gritar y removerme con gesto de incomodidad, sino decir «me duele». La sensación interna no posee una vida independiente de los criterios externos, no tiene por qué existir, como el escarabajo de la caja (PI, I, 293). Yo no me quejo porque tenga una sensación de dolor, ni siquiera porque siento dolor, sino porque me duele. La sensación, en definitiva, no es más que un término de un juego de lenguaje, el de las sensaciones; una forma de representación que podría ser sustituida por otra, de la que de momento no disponemos. Cuando Chihara y Fodor (1967, p. 173 y sig.) advierten que para Wittgenstein la relación entre conducta y mente se produce a través de un criterio son deudores, como veremos, de la tradición cartesiana.

Decíamos páginas atrás que no es que no exista la mentira, pero que para mentir necesitamos ya de la existencia de todo un juego de lenguaje. Supongamos que alguien se encuentra en el estado mental de "estar deprimido", este 


\section{2}

estado abarca una serie de aspectos, unos externos: enlentecimiento de movimientos, expresiones de tristeza, llantos, incapacitación laboral, etc., y otros internos: pensamientos de autodevaluación, culpa, ideas de suicidio, etc. Cuando hablamos de «estado mental de depresión» deberíamos referirnos a las dos categorías de entidades, frente a la tendencia a identificar el estado con los aspectos internos. Supongamos ahora que esa persona, por razones muy concretas, no quiere alarmar a su familia, quizá porque teme que le internen en una institución. En consecuencia cumple con los mínimos laborales, habla sólo de fútbol y pone buen cuidado en llorar cuando nadie le ve ni le puede reprochar sus ojos enrojecidos, aunque sigue manteniendo, exclusivamente para sí mismo, pensamientos de autodevaluación e ideas suicidas. El clínico dudaría de que aquí se tratara de una «auténtica» depresión, pues no cumple los criterios. Nosotros nos preguntamos si se trata de un estado depresivo sin conductas depresivas o si, más bien, estamos ante una mezcla de dos estados: depresión (leve) y temor al internamiento. La segunda solución nos evitaría postular un estado mental sin manifestaciones conductuales.

Dos comentarios al margen. Si el clínico tiene alguna noción de los pensamientos autodevaluatorios y de las ideas de suicidio es evidentemente porque muchos enfermos depresivos los relatan. Por otra parte, si se consigue que un enfermo depresivo vaya a trabajar, se mueva más y no hable de cosas tristes es muy probable que, por añadidura, mejoren los aspectos interiores (cogniciones).

Chihara y Fodor (1967, pp. 182-184) proclaman que los criterios se establecen en base a alguna característica observable del estado de cosas (state of affairs) y que se apoyan en inducciones a partir de correlaciones observadas. Recogen la frase ya citada de «Un "proceso interno" necesita criterios externos» (PI, I; 580) para atribuir a Wittgenstein una postura «conductista». Pero Wittgenstein en ningún momento utiliza el término «inducción». El que algo sea criterio de $\mathrm{X}$ no es cuestión de experiencia, sino de definición (PI, I, 354). Yo no induzco la existencia de lluvia a partir de mi observación de que caen gotas del cielo nublado, sino que es a eso mismo a lo que llamo «lluvia».

En cuanto a la observación de estos críticos de que para Wittgenstein los criterios conductuales son los únicos posibles, pensamos que el acento debe hacerse recaer en «únicos» y no en «posibles». Es cierto que el psicólogo observa los fenómenos de la vida mental (RPP, I, 286-292; PI, II, pp. 179-180) y que Wittgenstein entiende por «fenómeno» algo que puede ser observado (RPP, II, 75), luego el psicólogo sólo observa la conducta. Pero eso no quiere decir que el filósofo vienés sea conductista, como puedan serlo Skinner o el propio Ryle, pues él sólo acepta definiciones de los términos psicológicos que sean articuladas, frente a las definiciones operativas de los reduccionismos conductista y fisiológico (PR, 31, 32).

No se trata, por tanto, de que los términos mentales sean traducibles a términos conductuales, sino que o son términos conductuales o no son nada. Busquemos un ejemplo que nos ilumine. Para Wittgenstein el criterio de haber soñado es el sueño contado (PI, II, 184). Los niños aprenden lo que es soñar por medio del relato de alguien, incluso de ellos mismos, de las vivencias que han experimentado mientras se hallaban dormidos (RPP, I, 375, 376). Carece de sentido la pregunta de si alguien que cuenta un sueño lo ha tenido realmente o sufre un trastorno de memoria. Chihara y Fodor (1967, p. 185 y sig.) acusan al criterio de que conduce a sinsentidos. Por ejemplo, dicen, parecería que no tienen sentido frases como «Juan olvidó totalmente el sueño que tuvo la noche 
pasada». Pero, afirmamos, jes que realmente se trata de un sinsentido! Si Juan olvidó por completo su sueño no podemos siquiera especular sobre un sueño olvidado, no en mayor medida que en una paloma que vuela en el vacío, o en el puchero de monedas que se encuentra al final del arco iris. La pregunta de si esa proposición se entiende, o no, nos sirve de escasa ayuda, lo que tenemos que preguntarnos, siguiendo la inspiración wittgensteiniana (RPP, I, 366), es qué podemos hacer con ella.

Ahora bien, los científicos que han investigado el fenómeno del dormir hablan de los ritmos cerebrales alfa y beta y de los movimientos oculares rápidos (MOR) como criterios del sueño. Se ha descubierto cierta relación entre dichos fenómenos y el informe de los sujetos que, al ser despertados, afirmaban estar soñando. Pero, como ya señalaba Malcoln (1959) y, más recientemente, Chapman (1987), al adoptar nuevos criterios para la palabra «soñar» los científicos están modificando el concepto. $Y$ sus medidas, que no son otra cosa sino síntomas, no definen el sueño.

Las «definiciones operacionales» fueron utilizadas por primera vez por P. W. Bridgman (1927) en relación a la física. El concepto de longitud, por ejemplo, equivale exclusivamente a la serie de operaciones mediante las cuales se determina la longitud; un fenómeno no es más que un cojunto de operaciones, es sinónimo de ellas. Comentábamos antes que para Hempel (1954) este tipo de definición, cuando se especifica de forma precisa, está emparentada con el principio de verificación y, en cierta medida, comparte sus problemas. El principal de todos es la asistematicidad. El método operacional define el significado de un concepto o enunciado como la correspondencia con una operación o serie de operaciones, cuando la significación sólo es aplicable a sistemas teóricos y no a enunciados aislados.

El defensor más conspicuo del operacionalismo en psicología es, sin duda, B. F. Skinner (Cf. p. e. 1945, p. 159). Skinner reconoce que el punto de vista operacional tiene deficiencias - aunque no indica cuáles - pero que es bueno en todas las ciencias y especialmente en psicología: «debido a la presencia en este campo de un amplio vocabulario de origen antiguo y no científico» (1945, p. 161). Dicho vocabulario abarcaría a los términos mentalista, privados. Rechaza cualquier postulado o variable que se encuentre más allá de los datos observables del ambiente y de la conducta de los organismos. La variable externa de esta conducta es una función, de ahí el nombre de «análisis funcional» (Skinner, 1953, p. 23).

El rechazo de las variables internas se justifica por razones operativas. La deprivación de alimento, por ejemplo, puede ser medida y es, por tanto, un término operativo; cosa que no ocurre con la pulsión (drive) de anteriores teorías del aprendizaje (1953, p. 26). El conductismo radical, que niega la existencia de entidades subjetivas, es considerado por Skinner como la postura más adecuada (1976, p. 117).

Sin embargo, la gran paradoja del conductismo skinneriano, que nos descubre su filiación cartesiana, radica en que al negar la mente, identificándola exclusivamente con la entidades internas, niega un aspecto fundamental de la realidad que, como no podía ser menos, expulsado por la puerta retorna por la ventana. Al responder Skinner (1953, p. 262) a la pregunta de cómo aprendemos los términos verbales sobre nuestras intenciones dice que, primero, se enseña a la persona a utilizar estas palabras cuando exhibe la conducta pública adecuada. A partir de entonces, sigue diciendo, los estímulos privados son aso- 
ciados con las manifestaciones públicas (de los demás) y, desde entonces, la persona responde a los estímulos privados cuando ocurren sin manifestaciones públicas. «Estaba a punto de irme a casa» (was on the point of going bome) debe ser considerado, según Skinner, como el equivalente de «Observé acontecimientos en mí mismo que preceden, o acompañan, de forma característica mi marchar a casa.» Pero, le podemos objetar, anticipando en algo nuestra crítica a la teoría de James-Lange, que nadie toma una decisión porque observe que se producen en sí mismo cambios corporales. El método para encontrar el significado de las expresiones psicológicas no es mirar dentro del yo, sino examinar la función que juegan esas palabras y conceptos en nuestro lenguaje (PI, I, 413).

Los conductistas rechazan la introspección como método porque sus resultados no son públicamente verificables. Wittgenstein, en cambio, rechaza la introspección porque no es ningún método privilegiado de acceso a nuestros procesos subjetivos: «Puedo saber lo que el otro piensa, no lo que yo pienso» (PI, II, p. 222). Lo que yo pienso ni lo sé ni lo dejo de saber, y si hablamos de pensamiento inconsciente, en realidad estamos realizando un cambio conceptual, en el modo de representación, como hizo el psicoanálisis (Cf. Rodríguez Sutil, 1989).

Tanto el introspeccionismo como el conductismo comparten el supuesto cartesiano de que los procesos mentales sólo son directamente accesibles al sujeto que los experimenta y que, como destaca el ALP, dicho conocimiento es previo al aprendizaje del lenguaje público. Para Wittgenstein, en cambio, en la medida en que las experiencias subjetivas pueden ser expresadas de forma inteligible, existen criterios convencionales para identificarlas (Cf. Chapman, 1987, p. 111). $\mathrm{Si}$ existen criterios convencionales, esas experiencias son tan accesibles al psicólogo como al propio sujeto. Si no son expresables, son, por definición, inefables y caen en el ámbito de lo místico, aquello de lo que no podemos hablar, conclusión ya recogida en el Tractatus.

\section{VERIFICACIONISMO FISIOLOGICO}

Stephen Tulmin (1970, p. 22 y sig.) ha propuesto en un tono humorístico la «paradoja de Townes», en «homenaje» al neurólogo norteamericano Charles Twones, quien en una reunión celebrada en Colorado, allá por 1966, proclamaba con vehemencia que pronto sería posible explicar en términos neurofisiológicos todas las interconexiones e influencias del sistema nervioso central, y que así se podría explicar plenamente la conducta de las personas desde un punto de vista científico. La paradoja consiste en que los mismos científicos que proclaman lo anterior se reservan su responsabilidad personal y sienten orgullo justificado por sus propios pensamientos e ideas, cuando en realidad la teoría que defienden mantiene que todos sus actos vienen determinados.

La explicación biológica posee el indudable atractivo de remitirnos a un campo científico de mayor solidez que el psicológico. La biología se ajusta mejor al modelo del monismo materialista querido por la ciencia occidental. El reduccionismo neurofisiológico extremo ha sido defendido, entre otros, por D. O. Hebb (1949), para quien la «mente» sólo puede ser considerada con propósitos científicos como la activación del cerebro, y, más recientemente por $M$. Bunge (1980) y su materialismo emergentista, para quien el cerebro es un sistema con propiedades emergentes (no explicables por la física) como son las capacidades de percibir, sentir, recordar, imaginar, desear, pensar, etc. Sin embargo, el reduc- 
cionismo biológico también puede ser rastreado en posiciones que defiendan alguna forma de innatismo; innatismo de estructuras, como en el constructivismo piagetiano, pero, especialmente, de conocimientos, como en el generativismo chomskyano (Cf. Rodríguez Sutil, 1992).

Skinner (1950) con toda probabilidad está en lo cierto cuando afirma que el fisiologismo no nos ayuda mucho cuando intenta explicar la conducta recurriendo a fenómenos de otro nivel, por lo menos igual de complicados, descritos en términos diferentes (con otros términos operativos). Ahora bien, nuestro objetivo no es desechar la base fisiológica del comportamiento, lo que sería absurdo, sino situarla en su justa dimensión.

Los problemas mentales y los procesos cerebrales pertenecen en opinión de Wittgenstein a juegos de lenguaje diferentes:

La gramática de un estado o de un proceso mental es realmente, en muchos sentidos, similar a la de, por ejemplo, los procesos cerebrales. La principal diferencia es, quizá, que en el caso del proceso cerebral se admite como posible una comprobación directa; el proceso, en cuestión, puede tal vez ser visto abriendo el cráneo. Pero no hay lugar para una «percepción inmediata» similar en la gramática de los procesos mentales. (No existe tal movimiento en este juego.) (PG, 82)

Cabría interpretar un deseo, intención o expectativa como una inervación particular en el cerebro, pero la inervación como tal no es nada que quede abierto y necesitado de completamiento (PG, 132). Wittgenstein comienza diciendo que ninguna suposición le parece más natural que el que no existe ninguna razón para suponer que nuestros pensamientos proceden del cerebro y no del caos, de la misma forma que nada en la semilla se corresponde con la planta que surgirá de ella (RPP, I, 903; Z, 608). Es posible, por tanto, que ciertos fenómenos psicológicos no puedan ser investigados fisiológicamente, porque fisiológicamente nada les corresponde (RPP, I, 904; Z, 609; Cf. PI, I, 412).

El prejuicio en favor del paralelismo psicofísico es fruto de la concepción primitiva de la gramática. Se piensa que si admitimos una causalidad entre fenómenos psicológicos, no reductible a fenómenos fisiológicos, estamos admitiendo la existencia de un alma pegada al cuerpo (RPP, I, 906; Z, 611). No obstante, señala Hunter (1977, p. 515), Wittgenstein no niega que exista algo en el sistema nervioso responsable de los procesos psicológicos (CF. RPP, I, 907; $\mathrm{Z}, 612$ ). Lo que sí es improbable es que pensara que los procesos neuronales responsables de los movimientos de los labios y de la lengua cuando hablamos sean responsables también del hecho de que digamos esto en lugar de aquello.

De lo anterior podemos extraer la conclusión de que la psicología debe ocuparse, por definición, de lo que decimos y hacemos, como fenómenos articulados. Si se interesa por la fisiología es como ciencia que permite determinar los límites de lo que somos y de lo que no somos capaces de decir y hacer. El cerebro, vendríamos a decir, es un instrumento de la mente, entendida como contexto pragmático interpersonal, como también lo es la mano o el hígado. El cerebro cobra importancia, para la psicología, cuando está alterado, porque entonces se aparece de forma palmaria como límite. Cuando, en cambio, se identifica neurofisiología con psicología se cae en poder de un prejuicio cientifista, derivado de la imagen errónea de que pensamos con nuestras cabezas.

La ciencia que se expresa en prejuicios se transforma en metafísica de la peor calaña. Una idea científica es la que está siempre sujeta a discusión pues se refiere a la realidad empírica, es decir, a aquello que nos podemos imaginar de otra manera. Una convicción, en cambio, pertenece al ámbito de la gramáti- 
ca, al sistema de representación, es algo más difícil de modificar, y cuando se modifica no es ante la presencia de meros razonamientos sino mediante alguna forma de persuasión.

El papel central que desde hace siglos se concede al cerebro en los procesos de pensamiento, con ser la hipótesis más plausible, nunca debe ser tomado como una certeza absoluta. Recordemos, si no, la anécdota, recogida por Benjamin Farrington (1969, p. 135), del gran Aristóteles rechazando la doctrina de Alcmeón, 150 años anterior, de que el cerebro era la sede de las sensaciones, y prefiriendo el corazón como órgano sensible. Dicho rechazo, contra lo que pudiera pensarse, no procedía del prejuicio religioso o de la simple ignorancia, sino que se apoyaba en razones, por aquel entonces, bien fundamentadas, esto es, científicas. Entre esas razones habría que contar, por ejemplo, con que el cerebro es insensible a la estimulación directa; en los invertebrados muchas veces no se distinguen los ganglios cerebrales; sólo las partes con sangre son sensitivas y el cerebro (según decía Hipócrates) no tiene sangre; por otra parte, el Estagirita, en sus observaciones empíricas, no descubrió conexiones entre el cerebro y los órganos de los sentidos, cosa que sí ocurre con el corazón, centro del sistema vascular y del calor vital, etc.

En conclusión, los procesos psicológicos de una persona no son procesos en su cerebro sino lo que esa persona hace y dice en el contexto humano. $\mathrm{Y}$ eso es así aunque aceptemos que, al mismo tiempo, se producen inervaciones en su sistema nervioso y modificaciones en su sistema glandular necesarias, como prueban las investigaciones científicas, para que se desarrollen los comportamientos.

\section{LA TEORIA DE JAMES-LANGE SOBRE LAS EMOCIONES}

En su reciente libro sobre la filosofía de la psicología wittgensteiniana, Malcolm Budd (1989, p. 151) plantea que el objetivo principal de sus observaciones sobre la emoción era censurar la teoría de James-Lange. El famoso psicólogo y filósofo pragmatista norteamericano William James, hermano del no menos famoso escritor Henry James, escribía en su artículo original de 1884:

Nuestra manera de pensar sobre estas emociones estándar es que la percepción mental de algún hecho provoca la disposición mental llamada emoción y que este estado mental da lugar a la expresión corporal. Mi tesis, por el contrario, es que los cambios corporales siguen directamente a la percepción del becho desencadenante y que nuestra sensación de esos cambios según se van produciendo es la emoción. (p. 59)

Según la formulación sintética del propio James «no lloramos porque estamos tristes, sino que estamos tristes porque lloramos». Los cambios corporales son el fundamento de la emoción:

Si los estados corporales no siguieran a la percepción, esta última poseería una conformación totalmente cognitiva, pálida, incolora, carente de calor emocional. Entonces podríamos ver el oso y juzgar que lo mejor es correr, recibir la ofensa y considerar que lo correcto es golpear, pero no podríamos sentimos realmente asustados o iracundos. (id.)

Los cambios viscerales son imprescindibles para la emoción y, por tanto, deben existir patrones específicos para las distintas emociones. James suponía además que la producción voluntaria de los cambios viscerales de una emoción concreta deberían producir dicha emoción pero que no poseemos control vo- 
luntario sobre las vísceras. Esta hipótesis, como han mostrado Fernández Dols y Ortega (1985, p. 36), no se comprobó en experimentos posteriores.

La famosa crítica a James de Walter Cannon (1927) apunta, en esencia, a que la producción artificial de los cambios viscerales característicos de ciertas emociones no provoca por sí misma los efectos previstos. Nuestro Gregorio Marañón (1924) ya había dado apoyo a esa idea con un experimento que consistió en inyectar adrenalina a varios grupos de pacientes: sólo experimentan ansiedad aquellos que se encuentran especialmente predispuestos. Schachter y Singer (1962) trabajaron, años después, con una metodología similar. Estos autores sometieron a sujetos normales, inyectados con adrenalina o con un producto placebo, a diversas situaciones de interacción personal con un cómplice del examinador. Los resultados llevan a postular una teoría interactiva de dos factores: a) una activación fisiológica indiferenciada, y b) una evaluación cognitiva de las situaciones. Dadas las mismas circunstancias cognitivas el sujeto reacciona emocionalmente sólo si experimenta la activación fisiológica correspondiente. Esto podría considerarse una confirmación (moderada) de las tesis de James.

La maraña de estudios posteriores, según reconocen Fernández Dols y Ortega (1985, pp. 42-3) parecen debilitar la postura de James-Lange o la de Cannon, pero son, en sus propias palabras «exquisitamente inconcluyentes». La razón de dicha ambigüedad sólo proviene de que lo que aquí nos acecha es una problemática de tipo conceptual, irresoluble por cualquier prueba empírica.

La emoción, como ocurría con la intención y el deseo, es un término articulado del lenguaje y no tanto un concepto fenoménico, aunque aparezca asociada con síntomas corporales. Por tanto, las emociones no deberían aplicarse sin restricción a las criaturas sin lenguaje. Decimos, por ejemplo, que un perro teme que su dueño vaya a pegarle, pero no que le pegue mañana (PI, I, 650). Sólo quien domina el lenguaje puede tener sentimientos relacionados con la esperanza (PI, II, p. 174).

Ahora bien, si los conceptos referidos a las emociones son legítimamente aplicables a los animales, y parece que el uso así lo aconseja, tendremos que postular que la emoción engloba numerosos matices, algunos de ellos a caballo entre lo gramático y lo empírico. Lo empírico serían los síntomas corporales. Pero lo específicamente humano sería su carácter articulado.

Nos ha sorprendido agradablemente tener noticia de un trabajo del psicólogo ruso Lev S. Vygotsky (1984; Cf. Van der Veer, 1987) sobre la teoría de JamesLange que comparte y, al mismo tiempo, aclara algunas ideas de Wittgenstein. Vygotsky se plantea un ataque a la teoría mediante el análisis en profundidad de sus fundamentos metafísicos: el dualismo cartesiano. Descartes, en $\mathrm{Las} \mathrm{Pa}$ siones del Alma, intenta explicar la naturaleza de las pasiones como un «filósofo natural» y comienza describiendo el proceso corporal que da lugar a la emoción. Emociones, como sensaciones, dependen de los nervios que están conectados con el cerebro y que son como pequeños tubos por los que circulan los «espíritus animales», entidades a medio camino entre lo material y lo espiritual. Cuando una persona ve un objeto amenazador, los espíritus animales de los órganos de los sentidos se desplazan hasta el cerebro, donde interactúan con el alma, a través de la glándula pineal, haciendo que los espíritus animales se dirijan a diferentes partes del cuerpo, como pueden ser los músculos.

Descartes - y James - atribuyen a las pasiones una naturaleza, valga la redundancia, «pasiva» y perceptual. El alma es un perceptor pasivo, un registrador de movimientos de la glándula pineal o, en el teoría de James, la emoción 


\section{8}

es la toma de conciencia de los cambios viscerales. En consecuencia, las emociones son inmutables y, en último término, innatas (Vygotsky, 1984, p. 273). Es difícil, por lo demás, investigar un desarrollo real de las emociones, pues los procesos fisiológicos de niños y adultos son probablemente los mismos. Esta es una conclusión interesante: la ausencia del concepto de «desarrollo» es una consecuencia del dualismo mente-cuerpo.

Pero ya hemos visto, con Wittgenstein, la incongruencia de atribuir una cualidad exclusivamente mental a las emociones y, a la inversa, lo inadecuado de postular emociones humanas adultas en organismos no lingüísticos.

Los psicólogos subjetivistas y los objetivistas comparten, según Vygotsky (1984, p. 294) la misma concepción inadecuada de la explicación causal. Los primeros estudian los procesos psicológicos superiores, considerándolos indeterminados y libres como el alma de Descartes, mientras que los segundos se limitan al estudio de los procesos simples de estímulo-respuesta. Para Vygotsky y la escuela de Moscú, el medio primero y más importante del determinismo sociocultural de la conducta es el lenguaje (Van der Veer, 1987, p. 98). Respecto a las emociones, la idea más fecunda que nos presta esta escuela consiste en plantear la existencia de una evolución previa al lenguaje y otra posterior a la eclosión del mismo.

La emoción, por tanto, es un concepto intermedio entre lo empírico y lo gramático y, además, debemos afirmar que la persona actúa como totalidad en su entorno, del que forma parte.

\section{CONCLUSION}

Los reduccionismos, en su intento por superar la mitología introspectiva, caen en la propia trampa de aquello que pretenden negar, convirtiéndose, según la expresión de Vygotsky (1924), en un «idealismo vuelto del revés». Así, tanto conductistas como fisiologistas, defensores acérrimos del verificacionismo, al negar la conciencia expulsan el aspecto más definitorio del comportamiento humano, que podemos denominar «comunicación simbólica», o lo «reducen» a un ámbito con el cual es, por principio, inconmensurable: le inervación neuronal o la contracción muscular. Y las teorías intermedias, como la de James-Lange, enfrentan la dificultad insalvable de explicar de manera convincente el paralelismo psicofísico, problema originado en la división cartesiana de dos sustancias.

Ante esto Wittgenstein nos enseña que fenómeno y sentido no son dos realidades que se muestren por separado, sino que son abstracciones a partir de una misma presencia, dentro de un contexto social, es decir, lingüístico. La «persona» es un concepto primitivo, sólo por abstracción llegamos a plantearnos la existencia de dos realidades separadas: mente y cuerpo. Si se parte de esta separación como algo ya dado desde el principio sólo se alcanza la confusión, sobre todo cuando se localiza la mente en un espacio interior y oculto, accesible sólo por inferencia o introspección. Ese es el error en el que incurren en psicología tanto los reduccionismos como las posturas intermedias, interactivas. Al mostrar esto no se resuelven los problemas que esas teorías planteaban sino que se disuelven, al mostrar su naturaleza puramente verbal.

Para terminar afirmaremos que el comportamiento de las personas, como el de los animales, no es separable de su medio, pero ese medio, a diferencia de los animales, es social-comunicacional. Ese comportamiento tiene unos límites físicos (ecológicos) y biológicos (psicofisiológicos). 
Nota

1. Véanse al final del texto las abreviaturas sobre las obras de Wittgenstein citadas.

\section{ABREVIATURAS DE LAS OBRAS DE WITTGENSTEIN UTILIZADAS EN EL TEXTO}

T Tractatus Logico-Philosophicus (1918). Edición bilingüe alemán-español de Jacobo Muñoz e Isidoro Reguera; Madrid: Alianza, 1987.

PR Pbilosophical Remarks (1930). Se cita indicando la página de la edición inglesa de Raymond Hargreaves y Roger White; Oxford: Basil Blackwell, 1975.

PG Pbilosopbical Grammar (1932). Citamos indicando la página de la traducción inglesa de Anthony Kenny; Oxford: Basil Blackwell, 1974.

BIB-BrB The Blue and Brown Books (1933-35). Se cita indicando la página del original inglés; Oxford: Basil Blackwell, 1964. Traducción castellana de Francisco García Guillén «Los Cuadernos Azul y Marrón»; Madrid: Tecnos, 1984.

PI Pbilosophical Investigations (1945-49). La parte I se cita indicando el número de parágrafo; la parte II se cita según la paginación de la traducción inglesa de G. E. M. Anscombe; Oxford: Basil Blackweell, 1984. Edición bilingüe alemán-español de Alfonso García Suárez y Ulises Moulines «Investigaciones Filosóficas»; Barcelona: Crítica, 1988.

Z Zettel (1945-48). Citamos indicando el número de parágrafo. Traducción inglesa de G. E. M. Anscombe; Oxford: Basil Blackwell, 1981.

RPP Remarks on the Pbilosophy of Psychology (1945-49) (Vols. I y II). Se cita indicando el vol. y el número de parágrafo. Ediciones bilingües alemán-inglés, traducidas por G. E. M. Anscombe (I) y por C. G. Luckhardt y M. A. E. Aue (II); Oxford: Basil Blackwell, 1980.

\section{Referencias}

Bridgman, P. W. (1927). The Logic of Modern Pbysics. Nueva York: Macmillan.

Bruner, J. (1983). En Busca de la Mente. México: F. C. E.

Bund, M. (1989). Wittgenstein's Philosophy of Psychology. Londres: Routledge.

Bunge, M. (1980). The Mind-Body Problem. A Psychobiological Approach. Oxford: Pergamon.

Cannon, W. B. (1927). The James-Lange theory of emotions. A critical examination and an alternative theory. American Journal of Psychology, 39, 106-124.

Carnap, R. (1932-33). Psicología en Lenguaje Fisicalista. En A. J. Ayer (Ed.) (1959). El Positivismo Lógico; Madrid: FCE, 1965.

Chapman, M. (1987). Inner Processes and Outward Criteria: Wittgenstein's Importance for Psychology. En M. Chapman y R. A. Dixon (Eds.) (1987). Meaning and the Growth of Understanding. Wittgenstein's Significance for Developmental Psychology. Berlín: Springer.

Chinara, C. S., y Fodor, J. A. (1960). Operationalism and Ordinary Language: A Critique of Wittgenstein. En H. Morick (Ed.) (1967). Wittgenstein and The Problem of Other Minds. Nueva York: McGraw-Hill.

Descartes, R. Meditaciones Metafísicas. Con Objeciones y Respuestas. Traducción de Vidal Peña; Madrid: Alfaguara, 1977.

Descartes, R. Las Pasiones del Alma. Barcelona: Península, 1972. 
Farrington, B. (1969). Ciencia y Filosofía en la Antigüedad. Barcelona: Ariel, 1984.

Fernández Dols, J. M. y Ortega, J. E. (1985). Los Niveles de Análisis de la Emoción: James, cien años después. Estudios de Psicología, 21, 35-56.

García Sú́rez, A. (1976). La Lógica de la Experiencia. Madrid: Tecnos.

HeBB, D. O. (1949). The Organization of Behavior. Nueva York: Wiley.

Hempel, C. G. (1954). A Logical Appraisal of Operationalism. Scientifical Monthly, 79 , $215-220$

HunTer, J. F. M. (1977). Wittgenstein and Materialism. Mind, 86, 514-531.

James, W. (1884). ¿Qué es una emoción? Estudios de Psicología, 21, 57-73, 1985.

James, W. (1907). Pragmatismo. Buenos Aires: Aguilar, 1975.

Janik, A., y Toulmin, S. (1973). La Viena de Wittgenstein. Traducción de Ignacio Gómez de Liaño; Madrid: Taurus, 1974.

Kenny, A. (1972). Wittgenstein. Traducción de Alfredo Deaño; Madrid: Alianza, 1984.

KRAFT, V. (1950). Der Wiener Kreis. Viena: Springer.

MaLColm, N. (1959). The Concept of Dreaming. En H. Morick (Ed.) (1967). Wittgenstein and The Problem of Other Minds. Nueva York: McGraw-Hill.

Marañón, G. (1924). Contribución al estudio de la acción emotiva de la adrenalina. Estudios de Psicología, 21, 75-89, 1985.

Pole, D. (1958). La Ultima Filosofía de Wittgenstein. En Las Filosofias de Ludwig Wittgenstein, Barcelona: Oikos-Tau, 1966.

Rodríguez Sutit, C. (1989). Wittgenstein, discípulo de Freud y crítico del psicoanálisis. Clinica y Análisis Grupal, 11 (3), 52, 477-495.

Rodríguez SutiL, C. (1992). El Lenguaje del Pensamiento como Lenguaje Privado. Una Crítica Wittgensteiniana al Innatismo de Fodor. Psicothema, 4.

RYLE, G. (1949). The Concept of Mind. Nueva York: Barnes \& Noble.

Schachter, S., y Singer, J. (1962). Cognitive, social and physiological determinants of emotional state. Psychological Review, 59, 379-399.

Skinner, B. F. (1945). The Operational Analysis of Psychological Terms. Psychological Review, 52, 270-277 y 291-294.

Skinner, B. F. (1950). Are Theories of Learning Necessary? Psychological Review, 57, 193-216.

SkinNer, B. F. (1953). Science and Human Behavior. Nueva York: Macmillan.

Skinner, B. F. (1976). Particulars of my Life. Nueva York: Knopf.

Suppe, F. (1974). La Estructura de las Teorias Cientificas. Madrid: Editora Nacional, 1979.

Toulmin, S. (1970). Razones y Causas. En R. Borger y F. Cioffi (Eds.). La Explicación en las Ciencias de la Conducta. Madrid: Alianza, 1982.

Usom, J. O. (1956). Philosophical Analysis. Oxford University Press.

Veer, R. Van Der (1987). El Dualismo en Psicología: Un Análisis Vygotskyano. En M. Siguán (Ed.). Actualidad de Lev S. Vygotsky. Barcelona: Anthropos.

VyGotsky, L. S. (1924). El Método de Investigación Reflexológica y Psicológica. Infancia y Aprendizaje, 27/28, 87-104, 1984

VyGotsky, L. S. (1984). Sobranie socinenij. Moscú: Pedagógica. Citado por Van der Veer (1987).

VyGotsky, L. S. (1989). Concrete Human Psychology. Soviet Psychology, 27, 53-77. 